\title{
Lattice Gas Models in Contact with Stochastic Reservoirs: Local Equilibrium and Relaxation to the Steady State*
}

\author{
Gregory Eyink $^{1,2}$, Joel L. Lebowitz ${ }^{1}$ and Herbert Spohn ${ }^{2}$ \\ 1 Departments of Mathematics and Physics, Rutgers University, New Brunswick, NJ 08903, USA \\ 2 Theoretische Physik, Universität München, W-8000 München, Federal Republic of Germany
}

Received October 1, 1990

\begin{abstract}
Extending the results of a previous work, we consider a class of discrete lattice gas models in a finite interval whose bulk dynamics consists of stochastic exchanges which conserve the particle number, and with stochastic dynamics at the boundaries chosen to model infinite particle reservoirs at fixed chemical potentials. We establish here the local equilibrium structure of the stationary measures for these models. Further, we prove as a law of large numbers that the time-dependent empirical density field converges to a deterministic limit process which is the solution of the initial-boundary value problem for a nonlinear diffusion equation.
\end{abstract}

\section{Introduction}

We continue our study of the hydrodynamics of stochastic lattice gas models in a finite interval, with stochastic dynamics at the boundaries chosen to model the interaction with infinite particles reservoirs at fixed chemical potentials, which we began in our earlier paper [ELS] (hereafter referred to as I). We remind the reader that the model under consideration is a continuous-time Markov process on the finite state space $\Omega=\{0,1\}^{\Lambda}$, where $\Lambda=[-M, M] \cap \mathbb{Z}$ is a lattice of $(2 M+1)$ sites. The components $\eta_{x}, x \in \Lambda$ of the state vector $\eta \in \Omega$ denote the occupation numbers of the sites $x(1=$ occupied, $0=$ vacant $)$. We refer the reader to $I$ for an explicit definition of the stochastic dynamics, only pointing out here that finite-range, translation-invariant, non-degenerate rates are required, satisfying local detailed balance conditions and the technical "gradient" condition.

* Supported in part by NSF Grants DMR89-18903 and INT85-21407. G.E. and H.S. also supported by the Deutsche Forschungsgemeinschaft 
In I we studied the unique stationary measure of the lattice gas for large $M$ on a hydrodynamic scale. In particular, we proved that the empirical density field obeys a law of large numbers with respect to the stationary measures $\mu_{s s}^{\varepsilon}\left(\varepsilon=M^{-1}\right)$, converging weakly as $\varepsilon \rightarrow 0$ to a deterministic limit, which is given as the solution of a stationary transport equation. More generally, we showed that every extensive field defined by a bounded, local quantity obeys such a law of large numbers, converging to a deterministic limit which is an appropriate function of the local density. The proofs of these assertions were based on an entropy production argument of Guo, Papanicolaou and Varadhan [GPV], adapted to the situation with stochastic boundary dynamics.

We improve the analysis of the stationary measure, by showing that the strongest result obtainable by the GPV type of argument is a pointwise version of the local equilibrium property (LEP) for the stationary measure $\mu_{s s}^{\varepsilon}$ (a stronger result than the $L^{2}$ version we claimed in I). By this we mean the assertion that for any bounded, local function $g(\eta)$,

$$
\lim _{\varepsilon \rightarrow 0} \mu_{s s}^{\varepsilon}\left(g_{\llbracket \varepsilon^{-1} q \rrbracket}\right)=\langle g\rangle_{\bar{\rho}(q)},
$$

for every $q \in(-1,1)$, where $\bar{\rho}$ is the solution of the stationary transport equation with boundary conditions $\rho( \pm 1)=\rho\left(\lambda_{ \pm}\right)=\rho_{ \pm}$and $\langle\cdot\rangle_{\rho}$ is the expectation with respect to the infinite-volume Gibbs measure (for Hamiltonian $H$ ) at density $\rho$. Therefore, the stationary measures have the property that the expectations of any local function at a fixed position $q$ on the macroscopic scale converge to the corresponding equilibrium expectations with the local value of the density $\rho(q)$. The original argument of GPV does not yield this result, since their proof depended upon establishing small entropy production for space-time averaged measures. Our argument relies upon having sufficiently good local bounds on the entropy production (i.e. bounds on the entropy production of marginal distributions in local blocks, going to zero as $\varepsilon \rightarrow 0$ ) which follow automatically in the one-dimensional case from the global entropy bound and monotonicity of the entropy production. A further element of our proof is a local form of the two-block estimate of I which permits one to "tie together" neighboring blocks. Local equilibrium was obtained previously for the special case of symmetric simple exclusion dynamics by a duality argument [GKMP].

Next, we turn our attention to the time-dependent behavior of our stochastic dynamical system. In particular, we study the relaxation of initial local equilibrium distributions to the final steady state on the hydrodynamic scale. The goal here is to verify that the time-dependent empirical density field is given, in the hydrodynamic limit, by the solution of the initial-boundary value problem for the nonlinear diffusion equation: that is, by $\rho(q, \tau)$ which solves

$$
\begin{aligned}
\partial_{\tau} \rho(q, \tau) & =\partial_{q}\left[D(\rho(q, \tau)) \partial_{q} \rho(q, \tau)\right], \quad(q, \tau) \in[-1,1] \times[0, T], \\
\rho(q, 0) & =\rho_{0}(q), \quad q \in[-1,1], \\
\rho( \pm 1, \tau) & =\rho_{ \pm}, \quad \tau \in[0, T]
\end{aligned}
$$

for specified initial data $\rho_{0}(q)$ and boundary values $\rho_{ \pm}=\rho\left(\lambda_{ \pm}\right)$. As in the static case, $D(\rho)$ is the bulk diffusion coefficient for the dynamics, given by a Green-Kubo formula (see [Sp, DIPP]). 
The proof of the local equilibrium property for the stationary measures is the content of Sect. 2. The hydrodynamic relaxation to the steady state is discussed in Sect. 3. We freely borrow notations and definitions from I (e.g. we refer the reader to Sect. 2 of I for the definition of entropy production for our models). The results of Sect. 2 of $I$, in particular the fundamental Proposition 1 and its consequence Lemma 4, are the core of our present arguments, as they were of the static law of large numbers in I. We also continue the numbering of propositions of $I$, for the ease of reference of the reader.

\section{Local Equilibrium Property of the Stationary Measures}

Our goal is to prove the local equilibrium property of the stationary measure in the following precise form:

Theorem 3. For any bounded, local function $g(\eta)$,

$$
\lim _{\varepsilon \rightarrow 0} \mu_{s s}^{\varepsilon}\left(g_{\llbracket \varepsilon^{-1} q \rrbracket}\right)=\langle g\rangle_{\bar{\rho}(q)}
$$

for all $q \in(-1,1)$ (where $\langle\cdot\rangle$ denotes expectation with respect to the infinite-volume Gibbs measure at density $\rho$ ). Further, for any $g$ supported in $\mathbb{Z}^{\mp}$, if $\langle\cdot\rangle_{\rho}^{\mp}$ denotes expectation with respect to the Gibbs measure on the semi-infinite domain $\mathbb{Z}^{\mp}$ with free boundary conditions and asymptotic density $\rho$,

$$
\lim _{\varepsilon \rightarrow 0} \mu_{s s}^{\varepsilon}\left(g_{ \pm M}\right)=\langle g\rangle_{\rho^{ \pm}}^{\mp} .
$$

Proof. We consider first the case (2.1), where we have the inequality

$$
\begin{aligned}
\left|\mu_{s s}^{\varepsilon}\left(g_{\llbracket \varepsilon^{-1} q \rrbracket}\right)-\langle g\rangle_{\bar{\rho}(q)}\right| \leqq & \left|\mu_{s s}^{\varepsilon}\left(g_{\llbracket \varepsilon^{-1} q \rrbracket}\right)-\mu_{s s}^{\varepsilon}\left(A\left(g,\left[\varepsilon^{-1}(q-l), \varepsilon^{-1}(q+l)\right]\right)\right)\right| \\
& +\left|\mu_{s s}^{\varepsilon}\left(A\left(g,\left[\varepsilon^{-1}(q-l), \varepsilon^{-1}(q+l)\right]\right)\right)-\frac{1}{2 l} \int_{q-l}^{q+l} d q^{\prime} \hat{g}\left(\bar{\rho}\left(q^{\prime}\right)\right)\right| \\
& +\left|\frac{1}{2 l} \int_{q-l}^{q+l} d q^{\prime} \hat{g}\left(\bar{\rho}\left(q^{\prime}\right)\right)-\hat{g}(\bar{\rho}(q))\right|
\end{aligned}
$$

and we employ the notation from I that

$$
A(g, B)=\frac{1}{\#(\Lambda \cap B)} \sum_{x \in \Lambda \cap B} g_{x}(\eta)
$$

for any interval $B$, and

$$
\hat{g}(\rho)=\langle g\rangle_{\rho}
$$

Clearly, for each $q \in(-1,1)$, the last term on the right-hand side of $(2.3)$ goes to zero as $l \rightarrow 0$. On the other hand, the middle term goes to zero as $\varepsilon \rightarrow 0$ by the weak convergence of the extensive functions, established in I, and the boundedness of $g(\eta)$ and, consequently, of the averages $A(g, B)$.

Finally, we prove for the first term that, for each $q \in(-1,1)$,

$$
\lim _{l \rightarrow 0} \lim _{\varepsilon \rightarrow 0}\left|\mu_{s s}^{\varepsilon}\left(g_{\llbracket \varepsilon^{-1} q \rrbracket}\right)-\mu_{s s}^{\varepsilon}\left(A\left(g,\left[\varepsilon^{-1}(q-l), \varepsilon^{-1}(q+l)\right]\right)\right)\right|=0,
$$


which yields the first statement of the theorem. To obtain this result we shall modify the strategy of the proof for Proposition 1 . We observe the inequality

$$
\begin{aligned}
& \left|\mu_{s s}^{\varepsilon}\left(g_{\llbracket \varepsilon^{-1} q \rrbracket}\right)-\mu_{s s}^{\varepsilon}\left(A\left(g,\left[\varepsilon^{-1}(q-l), \varepsilon^{-1}(q+l)\right]\right)\right)\right| \\
& \quad \leqq\left|\mu_{s s}^{\varepsilon}\left(g_{\llbracket \varepsilon^{-1} q \rrbracket}\right)-\mu_{s s}^{\varepsilon}\left(A\left(g, \bar{B}_{0}\right)\right)\right|+\frac{1}{2 L} \sum_{j=-L}^{L} \mu_{s s}^{\varepsilon}\left(\left|A\left(g, \bar{B}_{0}\right)-A\left(g, \bar{B}_{j}\right)\right|\right),
\end{aligned}
$$

where $\left[\varepsilon^{-1}(q-l), \varepsilon^{-1}(q+l)\right]=\bigcup_{j=-L}^{L} \bar{B}_{j}, L=\llbracket l / \varepsilon k \rrbracket$, and the $2 L \bar{B}_{j}$ 's are blocks of $k$ sites each, disjoint, with $\llbracket \varepsilon^{-1} q \rrbracket$ at the center of $\bar{B}_{0}$. We introduce also the blocks $B_{j}$ of $k-2 R$ sites, obtained by removing the $R$ border sites at the ends of each $\bar{B}_{j}(R$ the range of the interaction). We see that it suffices to prove

$$
\lim _{\varepsilon \rightarrow 0}\left|\mu_{s s}^{\varepsilon}\left(g_{\llbracket \varepsilon^{-1} q \rrbracket}\right)-\mu_{s s}^{\varepsilon}\left(A\left(g, B_{0}\right)\right)\right|=0,
$$

ii) $\quad \lim _{k \rightarrow \infty} \lim _{l \rightarrow 0} \lim _{\varepsilon \rightarrow 0} \sup _{j} \mu_{s s}^{\varepsilon}\left(\left|A\left(g, B_{0}\right)-A\left(g, B_{j}\right)\right|\right)=0$.

For i), we will use the fact that the limiting canonical Gibbs measures as $\varepsilon \rightarrow 0$ are translation-invariant (in the infinite-volume limit, i.e. as $k \rightarrow+\infty$ ). To make the argument precise, introduce a block of $r$ spins $B_{0}^{\prime} \supset B_{0}, r>k$, and let $\mu_{q, r}^{\varepsilon}$ be the marginal of $\mu_{s s}^{\varepsilon}$ on $B_{0}^{\prime}$, considered as a measure on configurations in a fixed interval $\left[-\frac{r}{2}, \frac{r}{2}\right]_{\mathbb{Z}}$. By the main entropy production bound and monotonicity,

$$
\sigma_{B_{0}^{\prime}}\left[\mu_{q, r}^{\varepsilon}\right] \leqq c \varepsilon .
$$

Hence, any weak limit point $\mu_{q, r}^{*}$ of $\mu_{q, r}^{\varepsilon}$ as $\varepsilon \rightarrow 0$ must be a convex combination of the canonical Gibbs measures $\mathscr{G}_{c}$ on $\left[-\frac{r}{2}, \frac{r}{2}\right]_{\mathbb{Z}}$. In particular,

$$
\begin{aligned}
\varlimsup_{\varepsilon \rightarrow 0}\left|\mu_{s s}^{\varepsilon}\left(g_{\llbracket \varepsilon^{-1} q \rrbracket}\right)-\mu_{s s}^{\varepsilon}\left(A\left(g, B_{0}\right)\right)\right| & =\varlimsup_{\varepsilon \rightarrow 0}\left|\mu_{q, r}^{\varepsilon}\left(g_{0}\right)-\mu_{q, r}^{\varepsilon}\left(A\left(g, B_{0}\right)\right)\right| \\
& \leqq \sup _{N=0, \ldots, r}\left|v_{N}\left(g_{0}\right)-v_{N}\left(A\left(g, B_{0}\right)\right)\right|,
\end{aligned}
$$

where $v_{N}$ is the canonical Gibbs measure on $B_{0}^{\prime}$ with $N$ particles (actually, we should also specify the fixed occupancies $\eta_{B}$ in the boundary regions of $B_{0}^{\prime}$ of width $R$, as $v_{N, \eta_{B}}$-for simplicity, we have neglected this.) Because, for each $r$, the sup is realized for some $N_{r}$ and since there is the bound $0 \leqq N_{r} / r \leqq 1$, a subsequence $\left(r_{j}\right)$ may be chosen so that $r_{j} \uparrow+\infty$ and

It follows that for each $k$,

$$
\frac{N_{r_{j}}}{r_{j}} \underset{j \rightarrow \infty}{\longrightarrow} \rho \in[0,1] \text {. }
$$

$$
\begin{aligned}
\varlimsup_{\varepsilon \rightarrow 0}\left|\mu_{s s}^{\varepsilon}\left(g_{\llbracket \varepsilon^{-1} q \rrbracket}\right)-\mu_{s s}^{\varepsilon}\left(A\left(g, B_{0}\right)\right)\right| & \leqq \lim _{j \rightarrow \infty}\left|v_{N_{r_{j}}}\left(g_{0}\right)-v_{N_{r_{j}}}\left(A\left(g, B_{0}\right)\right)\right| \\
& =\left|\left\langle g_{0}\right\rangle_{\rho}-\left\langle A\left(g, B_{0}\right)\right\rangle_{\rho}\right| \\
& =0,
\end{aligned}
$$

the latter by the translation-invariance of the Gibbs measure. This gives i). 
For ii), we employ a modified version of the argument for the two-block estimate in Theorem 1. For each $\varepsilon, l$ the supremum in (2.8) is achieved for some $j_{\varepsilon, l}$. For each $\varepsilon, l$ we define $\mu_{q, k}^{\varepsilon, l}$ to be the joint marginal of $\mu_{s s}^{\varepsilon}$ in the two blocks $B_{0}$ and $B_{j_{c, l}}$, considered as a measure on the fixed set $\{0,1\} \times\left[-\left(\frac{k}{2}-R\right), \frac{k}{2}-R\right]_{\mathbb{Z}}$ consisting of two copies of $\left[-\left(\frac{k}{2}-R\right), \frac{k}{2}-R\right]_{\mathbb{Z}}$, denoted $B_{0}$ and $B_{1}$. Define, then, as in Theorem 1, the Dirichlet form

$$
D_{01}(f)=\frac{1}{2 k^{2}} \sum_{x \in B_{0}} \sum_{y \in B_{1}}\left\langle\tilde{c}(x, y ; \eta)\left[f\left(\eta^{x y}\right)-f(\eta)\right]^{2}\right\rangle_{\rho}
$$

for $f$ the density of a measure on $B_{0} \cup B_{1}$. Furthermore, introduce

$$
\tilde{\sigma}_{B_{0} \cup B_{1}}[f]=\sigma_{B_{0} \cup B_{1}}[f]+D_{01}(\sqrt{f}) .
$$

As a consequence of the main entropy estimate and the Lemma 2 of I, we have that

$$
\tilde{\sigma}_{B_{0} \cup B_{1}}\left[f_{q, k}^{\varepsilon, l}\right] \leqq \text { const. } \varepsilon+\text { const. } l \text {. }
$$

Therefore, any limit point $f_{q, k}^{*}$ of $f_{q, k}^{\varepsilon, l}$ as $\varepsilon \rightarrow 0$ and then $l \rightarrow 0$ must have $\tilde{\sigma}_{B_{0} \cup B_{1}}\left[f_{q, k}^{*}\right]=0$. Note, however, that the functional $\tilde{\sigma}_{B_{0} \cup B_{1}}$ has the same essential properties for $B_{0} \cup B_{1}$ as $\sigma_{B_{0}}$ has for $B_{0}$ alone: it is a positive, convex function, which is strictly convex on the set of $f$ 's which have fixed occupancies on the boundary regions of each block and fixed total (combined) number of particles in the two boxes. Hence, there is a unique value $f_{\min }$ in that domain where the minimum value $\tilde{\sigma}_{B_{0} \cup B_{1}}\left[f_{\min }\right]=0$ is attained and it is easy to verify that $f_{\min }$ is the finite version of a canonical Gibbs measure on $B_{0} \cup B_{1}$ with specified occupancies outside. Therefore, the limit points $f_{q, k}^{*}$ are in the set of convex combinations of such extremal elements. Hence,

$$
\begin{aligned}
& \varlimsup_{l \rightarrow 0} \varlimsup_{\varepsilon \rightarrow 0} \sup _{j} \mu_{s s}^{\varepsilon}\left(\left|A\left(g, B_{0}\right)-A\left(g, B_{j}\right)\right|\right) \\
& \quad \leqq \sup _{N=0, \ldots, 2 k-4 R} v_{N}\left(\left|A\left(g, B_{0}\right)-A\left(g, B_{1}\right)\right|\right) .
\end{aligned}
$$

We may argue as before that for each $k$, the supremum is actually achieved for some $N_{k} \in\{0, \ldots, 2 k-4 R\}$ and then, since,

$$
0 \leqq \frac{N_{k}}{2 k} \leqq 1,
$$

along any subsequence for which $v_{N_{k}}\left(\left|A\left(g, B_{0}\right)-A\left(g, B_{1}\right)\right|\right)$ converges, a further subsequence $k_{j}$ may be chosen so that

$$
\frac{N_{k_{j}}}{2 k_{j}} \underset{j \rightarrow \infty}{\longrightarrow} \rho \in[0,1] .
$$

Then, by the $L^{1}$ law of large numbers for the canonical ensemble,

$$
\begin{aligned}
\lim _{j \rightarrow \infty} v_{N_{k_{j}}}\left(\left|A\left(g, B_{0}\right)-A\left(g, B_{1}\right)\right|\right) \leqq & \varlimsup_{j \rightarrow \infty} v_{N_{k_{j}}}\left(\left|A\left(g, B_{0}\right)-\hat{g}(\rho)\right|\right) \\
& +\varlimsup_{j \rightarrow \infty} v_{N_{k_{j}}}\left(\left|A\left(g, B_{1}\right)-\hat{g}(\rho)\right|\right) \\
& =0
\end{aligned}
$$


so that the subsequence of $v_{N_{k}}\left(\left|A\left(g, B_{0}\right)-A\left(g, B_{1}\right)\right|\right)$ necessarily converges to zero. Thus,

which gives ii).

$$
\varlimsup_{k \rightarrow 0} \varlimsup_{l \rightarrow 0} \varlimsup_{\varepsilon \rightarrow 0} \sup _{j} \mu_{s s}^{\varepsilon}\left(\left|A\left(g, B_{0}\right)-A\left(g, B_{1}\right)\right|\right)=0,
$$

The second part of the theorem, embodied in (2.2), is somewhat easier to prove. Consider the right endpoint. We may write

$$
\mu_{s s}^{\varepsilon}\left(g_{M}\right)=\left\langle f_{+, r}^{\varepsilon} g\right\rangle_{\rho}
$$

for every $r$ for which supp $g \subseteq[-r, 0]$, where $f_{+, r}^{\varepsilon}$ is the density with respect to the Gibbs measure $v_{\rho}$ of the marginal of $\mu_{s s}^{\varepsilon}$ in the interval $[M-r-R, M]_{\mathbb{Z}}$, considered as a measure on configurations in a fixed interval $[-r-R, 0]_{\mathbb{Z}}$. In the proof of Proposition 1 in I, it was shown that, for each $r$, the limit $f_{+, r}^{*}$ of $f_{+, r}^{\varepsilon}$ as $\varepsilon \rightarrow 0$ exists and equals the density of the Gibbs measure at density $\rho_{+}$on the interval $[-r-R, 0]_{\mathbb{Z}}$, with free boundary conditions at the right endpoint and specified distribution in the left boundary interval $[-r-R,-r]_{\mathbb{Z}}$. Therefore,

$$
\lim _{\varepsilon \rightarrow 0} \mu_{s s}^{\varepsilon}\left(g_{M}\right)=\left\langle f_{+, r}^{*} g\right\rangle_{\rho} .
$$

Since $r$ is arbitrary, we may take subsequently $r \rightarrow+\infty$. Since the system of conditional distributions of $\mu_{+, r}^{*}$ converge in this limit to a consistent set of conditional distributions on the subsets of the semi-infinite interval $[-\infty, 0]_{\mathbb{Z}}$, satisfying the lattice DLR equations with chemical potential $\lambda\left(\rho_{+}\right)$and free boundary conditions at the right end, and since the Gibbs measure is unique in one dimension (e.g. see $[\mathrm{G}]$ ), it follows that

$$
\lim _{\varepsilon \rightarrow 0} \mu_{s s}^{\varepsilon}\left(g_{M}\right)=\langle g\rangle_{\rho_{+}}^{-} .
$$

This completes the proof of Theorem 3.

We remark that the local equilibrium property is a stronger result than the weak convergence of the extensive fields. A relatively simple argument using the Chebyshev inequality gives the convergence in probability to a deterministic limit, which, with tightness, gives the weak convergence (see [DIPP], Proposition 4.1). However, local equilibrium does not apparently follow from the weak convergence result alone (but, rather, as above, from its proof).

\section{Relaxation to the Steady State}

For the model introduced in I, we will now consider the stochastic process $\eta_{x, \varepsilon^{-2}{ }_{\tau}}, 0 \leqq \tau \leqq T, x=-\varepsilon^{-1}, \ldots, \varepsilon^{-1},\left(\varepsilon^{-1}=M\right)$ with the initial measures $\mu^{\varepsilon}$ required to satisfy for each $\delta>0$ and $\phi \in \mathscr{D}([-1,1])$,

$$
\lim _{\varepsilon \rightarrow 0} \mu^{\varepsilon}\left(\left|X_{0}^{\varepsilon}(\phi)-\int_{-1}^{1} d q \phi(q) \rho_{0}(q)\right|>\delta\right)=0
$$

for some fixed $\rho_{0} \in \mathscr{M}_{1}$, where, as in I, $\mathscr{M}_{1}=L_{1}^{\infty}([-1,1])$ is the set of non-negative measurable functions on $[-1,1]$ essentially bounded by one. In this section, $P^{\varepsilon}$ denotes the path measure for the process and $E^{\varepsilon}$ the corresponding expectation. 
We regard $\tau \mapsto \rho_{\tau}^{\varepsilon}$, with $\rho_{\tau}^{\varepsilon}(q) \equiv \eta_{\llbracket \varepsilon^{-1} q \rrbracket, \varepsilon^{-2} \tau}$, as an element of $D\left([0, T], \mathscr{M}_{1}\right)$, the space of right continuous trajectories. with left limits equipped with the Skohorod topology. The path measure $P^{\varepsilon}$ induces a path measure on $D\left([0, T], \mathscr{M}_{1}\right)$ : without risk of confusion, this measure is again denote by $P^{\varepsilon}$, expectations by $E^{\varepsilon}$.

We define $\rho \in L_{1}^{\infty}([-1,1] \times[0, T])$ to be a weak solution of the initial-boundary value problem $(4.1-3)$ if, for every $\phi \in C^{2}([-1,1])$ with $\phi( \pm 1)=0$ and a.e. $\tau \in[0, T]$,

$$
\begin{aligned}
& \int_{-1}^{1} d q \phi(q) \rho(q, \tau)-\int_{-1}^{1} d q \phi(q) \rho_{0}(q) \\
& \quad=\int_{0}^{\tau} d \sigma\left[\int_{-1}^{1} d q \phi^{\prime \prime}(q) \hat{h}(\rho(q, \sigma))-\phi^{\prime}(+1) \hat{h}\left(\rho_{+}\right)+\phi^{\prime}(-1) \hat{h}\left(\rho_{-}\right)\right] .
\end{aligned}
$$

For every $\rho_{0} \in \mathscr{M}_{1}$ and $\rho_{ \pm} \in[0,1]$ there exist solutions of (3.2), which are furthermore unique under an additional regularity assumption, such as $\rho \in L^{2}\left([0, T], H_{0}^{1}+\bar{\rho}\right)$ with $H_{0}^{1}$ the Sobolev space of functions with $L^{2}$ derivatives which vanish at $q= \pm 1$ (see below). Given $\rho_{0} \in \mathscr{M}_{1}$, we define the measure $P=\delta_{\rho}$ on $D\left([0, T], \mathscr{M}_{1}\right)$, where $\rho$ is the unique weak solution corresponding to initial data $\rho_{0}$.

The main result we claim in this section is:

Theorem 4. $P$ is the weak limit of $P^{\varepsilon}$ as $\varepsilon \rightarrow 0$.

As for the static result in I, the proof of Theorem 4 requires an estimate on entropy production which is provided by

Proposition 4. If $\mu^{\varepsilon}=f^{\varepsilon} v_{\rho}^{\varepsilon}$ is an arbitrary measure on $\{0,1\}^{\wedge}$ and $\bar{\mu}^{\varepsilon}$ is its time-average over the interval $\left[0, \varepsilon^{-2} \tau\right]$, with density given by

$$
\bar{f}^{\varepsilon}=\frac{1}{\varepsilon^{-2} \tau} \int_{0}^{\varepsilon^{-2} \tau} d t e^{L^{* t}} f^{\varepsilon},
$$

( $L^{*}$ denotes the adjoint of $L$ with respect to $v_{\rho}$ ), then, for $\sigma^{\varepsilon}\left[f^{\varepsilon}\right]$ the entropy production defined in $(\mathrm{I} ; 2.1)$,

$$
\sigma^{\varepsilon}\left[\bar{f}^{\varepsilon}\right] \leqq\left(\frac{2 c}{\tau}+c\right) \varepsilon
$$

for some $c>0$; in particular, $\bar{\mu}^{\varepsilon} \in S(\varepsilon)=\left\{f \mid \sigma^{\varepsilon}[f] \leqq c \varepsilon, f \geqq 0,\langle f\rangle_{\rho}^{\varepsilon}=1\right\}$.

This proposition allows again the application of the fundamental Proposition 1 and its consequence Lemma 4 of I to the present situation. The proof of Theorem 4 itself requires the verification of two key statements:

(1) $\left(P^{\varepsilon} \mid \varepsilon>0\right)$ is a tight family.

(2) If $P^{*}$ is any weak limit of the family as $\varepsilon \rightarrow 0$, then $P^{*}$ is supported by $C\left([0, T], \mathscr{M}_{1}\right) \cap L^{2}\left([0, T], H_{0}^{1}+\bar{\rho}\right)$ and for every $\phi \in C^{2}[-1,1]$ with $\phi( \pm 1)=0$ and every $\tau \in[0, T]$,

$$
\begin{aligned}
\int_{-1}^{1} d q \phi(q)\left[\rho(q, \tau)-\rho_{0}(q)\right] \\
\quad=\int_{0}^{\tau} d \sigma\left[\int_{-1}^{1} d q \phi^{\prime \prime}(q) \hat{h}(\rho(q, \sigma))-\phi^{\prime}(+1) \hat{h}\left(\rho_{+}\right)+\phi^{\prime}(-1) \hat{h}\left(\rho_{-}\right)\right] P^{*}-\text { a.s.. }
\end{aligned}
$$


The proof of Theorem 4 is then completed by showing that the weak solution given by (3.5) is unique under the stated regularity properties. The plan of the following is to first establish Proposition 4, then to prove the statements (1) and (2) for Theorem 4 and, finally, to conclude the proof of Theorem 4 by establishing the regularity and unicity of the profiles in the support of limiting $P^{*}$.

Proof of Proposition 4. We define the relative entropy

$$
H^{\varepsilon}\left[f^{\varepsilon}\right]=-\left\langle f^{\varepsilon} \log f^{\varepsilon}\right\rangle_{\rho}^{\varepsilon}+\sum_{x=-M}^{M} \lambda_{x}\left\langle\eta_{x} f^{\varepsilon}\right\rangle_{\rho}^{\varepsilon},
$$

where $\lambda_{x}=\frac{1}{2}\left(\lambda_{+}+\lambda_{-}\right)+\frac{1}{2}\left(\lambda_{+}-\lambda_{-}\right)(\varepsilon x)$ is the linear profile interpolating between $\lambda_{+}$at $x=+M$ and $\lambda_{-}$at $x=-M$. Observe that $H^{\varepsilon}$ is extensive in the sense that it satisfies a bound of the form

$$
\left|H^{\varepsilon}\left[f^{\varepsilon}\right]\right| \leqq c \cdot \varepsilon^{-1}
$$

for some $c>0$ uniformly for all $f^{\varepsilon}$. Furthermore, by particle conservation

$$
\begin{aligned}
\sum_{x=-M}^{M} \lambda_{x}\left\langle L \eta_{x}\right\rangle= & \frac{1}{2}\left(\lambda_{+}-\lambda_{-}\right) \varepsilon \sum_{x=-M}^{M}\left\langle j_{1}(x)\right\rangle+\lambda_{+}\left\langle j_{+}\right\rangle+\lambda_{-}\left\langle j_{-}\right\rangle \\
= & \frac{1}{2}\left(\lambda_{+}-\lambda_{-}\right) \varepsilon\left\langle\left(h_{-(M-R)}-h_{M-R+1}\right)\right\rangle \\
& +\frac{1}{2}\left(\lambda_{+}-\lambda_{-}\right) \varepsilon \sum_{x:|x \pm M| \leqq R}\left\langle j_{1}(x)\right\rangle \\
& +\lambda_{+}\left\langle j_{+}\right\rangle+\lambda_{-}\left\langle j_{-}\right\rangle,
\end{aligned}
$$

where the gradient condition (1.7) has been used in the second equality.

Let $f_{t}^{\varepsilon}=e^{L^{*} t} f^{\varepsilon}$. Then, with $f_{0}^{\varepsilon}=f^{\varepsilon}$,

$$
\begin{aligned}
\left.\frac{d}{d t} H^{\varepsilon}\left[f_{t}^{\varepsilon}\right]\right|_{t=0}= & \sigma^{\varepsilon}\left[f^{\varepsilon}\right]+\frac{1}{2}\left(\lambda_{+}-\lambda_{-}\right) \varepsilon\left\langle\left(h_{-(M-R)}-h_{M-R+1}\right) f^{\varepsilon}\right\rangle_{\rho}^{\varepsilon} \\
& +\frac{1}{2}\left(\lambda_{+}-\lambda_{-}\right) \varepsilon \sum_{x:|x \pm M| \leqq R}\left\langle j_{1}(x) f^{\varepsilon}\right\rangle_{\rho}^{\varepsilon},
\end{aligned}
$$

(compare with $(\mathrm{I} ; 3.16))$, so that

$$
\int_{0}^{t} \sigma^{\varepsilon}\left[f_{s}^{\varepsilon}\right] d s=H^{\varepsilon}\left[f_{t}^{\varepsilon}\right]-H^{\varepsilon}\left[f^{\varepsilon}\right]+O(\varepsilon \cdot t)
$$

by boundedness of $h_{x}$ and $j_{1}(x)$. Since the entropy production is convex,

$$
\sigma^{\varepsilon}\left[\bar{f}^{\varepsilon}\right] \leqq \frac{1}{t} \int_{0}^{t} d s \sigma^{\varepsilon}\left[f_{s}^{\varepsilon}\right]
$$

with $\bar{f}^{\varepsilon}$ the time-average over $[0, t]$ of $f^{\varepsilon}$. From (3.11), (3.10) and (3.7) it follows that

$$
\sigma^{\varepsilon}\left[\bar{f}^{\varepsilon}\right] \leqq \frac{2 c}{\varepsilon \cdot t}+c \cdot \varepsilon,
$$


and, in particular,

$$
\sigma^{\varepsilon}\left[\bar{f}^{\varepsilon}\right] \leqq\left(\frac{2 c}{\tau}+c\right) \varepsilon
$$

with $t=\varepsilon^{-2} \tau$. This is just the conclusion of Proposition 4 .

We now verify the key statements (1) and (2) for Theorem 4.

Ad (1): This can be accomplished by a standard machinery. We note that for $\phi \in \mathscr{D}([-1,1])$ and $\varepsilon$ sufficiently small (so that $\phi(\varepsilon x)=0$ for $|x \pm M| \leqq R$ )

$$
\begin{aligned}
\varepsilon^{-2} L X^{\varepsilon}(\phi) & =\varepsilon \sum_{x=-\varepsilon^{-1}}^{\varepsilon^{-1}} \varepsilon^{-2}(\phi(\varepsilon x+\varepsilon)+\phi(\varepsilon x-\varepsilon)-2 \phi(\varepsilon x)) h_{x}(\eta) \\
& =X^{\varepsilon}\left(h ; \phi^{\prime \prime}\right)+O(\varepsilon)
\end{aligned}
$$

and

$$
\begin{aligned}
\varepsilon^{-2}\left(L X^{\varepsilon}(\phi)^{2}-2 X^{\varepsilon}(\phi) \cdot L X^{\varepsilon}(\phi)\right) & =\sum_{x=-\varepsilon^{-1}}^{\varepsilon^{-1}}(\phi(\varepsilon x)-\phi(\varepsilon x+\varepsilon))^{2} c(x, x+1 ; \eta) \\
& =\varepsilon X^{\varepsilon}\left(c ;\left(\phi^{\prime}\right)^{2}\right)+O\left(\varepsilon^{2}\right) .
\end{aligned}
$$

Let

$$
X_{\tau}^{\varepsilon}(\phi)=\varepsilon \sum_{x=-\varepsilon^{-1}}^{\varepsilon^{-1}} \phi(\varepsilon x) \eta_{\varepsilon^{-2} \tau}(x)
$$

The associated martingale, $M_{\tau}^{\varepsilon}(\phi)$, is given by

$$
X_{\tau}^{\varepsilon}(\phi)-X_{0}^{\varepsilon}(\phi)=\int_{0}^{\tau} d \sigma \varepsilon^{-2} L X^{\varepsilon}(\phi)\left(\eta_{\varepsilon^{-2} \sigma}\right)+M_{\tau}^{\varepsilon}(\phi)
$$

with the quadratic variation

$$
E^{\varepsilon}\left(M_{\tau}^{\varepsilon}(\phi)^{2}\right)=\varepsilon^{-2} \int_{0}^{\tau} d \sigma E^{\varepsilon}\left(L X_{\sigma}^{\varepsilon}(\phi)^{2}-2 X_{\sigma}^{\varepsilon}(\phi) L X_{\sigma}^{\varepsilon}(\phi)\right)
$$

Let

$$
\gamma_{1, \tau}^{\varepsilon}(\phi)=\varepsilon^{-2} L X_{\tau}^{\varepsilon}(\phi)
$$

and

$$
\gamma_{2, \tau}^{\varepsilon}(\phi)=\varepsilon^{-2}\left(L X_{\tau}^{\varepsilon}(\phi)^{2}-2 X_{\tau}^{\varepsilon}(\phi) L X_{\tau}^{\varepsilon}(\phi)\right) .
$$

Then, by (3.14) and (3.15), there exists a constant $c$, independent of $\varepsilon$ and $\phi$, such that

$$
E^{\varepsilon}\left(\gamma_{1, \tau}^{\varepsilon}(\phi)^{2}\right) \leqq c \int_{-1}^{1} d q \phi^{\prime \prime}(q)^{2}
$$

and

$$
E^{\varepsilon}\left(\gamma_{2, \tau}^{\varepsilon}(\phi)^{2}\right) \leqq c \varepsilon^{2}\left(\int_{-1}^{1} d q \phi^{\prime}(q)^{2}\right)^{2}
$$

This implies tightness of the family $\left(P^{\varepsilon} \mid \varepsilon>0\right)$ [M, DIPP]. 
Furthermore, any weak limit $P^{*}$ is supported on the set of continuous trajectories $C\left([0, T], \mathscr{M}_{1}\right)$ because $X_{\tau}^{\varepsilon}(\phi)$ changes in a jump at most by $\left\|\phi^{\prime}\right\|_{\infty} \varepsilon^{2}$.

$\operatorname{Ad}(2)$ : It is useful here to consider the fields $X^{\varepsilon, k}(\phi)$ "cut-off" a finite microscopic distance $k$ from the boundaries:

$$
X^{\varepsilon, k}(\phi) \equiv \varepsilon \sum_{x=-\varepsilon^{-1}+k}^{\varepsilon^{-1}-k} \phi(\varepsilon x) \eta_{x} .
$$

If we consider $\phi \in C^{2}([-1,1])$ with $\phi( \pm 1)=0$ but permit $\phi^{\prime}( \pm 1) \neq 0$, then (3.14) must be replaced by

$$
\begin{aligned}
\varepsilon^{-2} L X^{\varepsilon, k}(\phi)= & \varepsilon \sum_{x=-\varepsilon^{-1}+k}^{\varepsilon^{-1}-k} \varepsilon^{-2}(\phi(\varepsilon x+\varepsilon)+\phi(\varepsilon x-\varepsilon)-2 \phi(\varepsilon x)) h_{x}(\eta) \\
& +\varepsilon^{-1}\left[\phi(1-\varepsilon k) h_{M-k+1}-\phi(1-\varepsilon k+\varepsilon) h_{M-k}\right] \\
& +\varepsilon^{-1}\left[\phi(-1+\varepsilon k) h_{-M+k-1}-\phi(-1+\varepsilon k-\varepsilon) h_{-M+k}\right] \\
= & X^{\varepsilon}\left(h ; \phi^{\prime \prime}\right)+\phi^{\prime}(1)\left[(k-1) h_{M-k}-k h_{M-k+1}\right] \\
& +\phi^{\prime}(-1)\left[k h_{-M+k-1}-(k-1) h_{-M+k}\right]+O(\varepsilon) .
\end{aligned}
$$

Defining as above

$$
\gamma_{2, \tau}^{\varepsilon, k}(\phi) \equiv \varepsilon^{-2}\left(L X_{\tau}^{\varepsilon, k}(\phi)^{2}-2 X_{\tau}^{\varepsilon, k}(\phi) \cdot L X_{\tau}^{\varepsilon, k}(\phi)\right),
$$

we find similarly the bound

$$
E^{\varepsilon}\left(\gamma_{2, \tau}^{\varepsilon, k}(\phi)^{2}\right) \leqq c \cdot \varepsilon^{2}\left(\int_{-1}^{1} d q \phi^{\prime}(q)^{2}\right)^{2} .
$$

It is important here to observe that this bound is independent of $k$. Since the quadratic variation of $M_{\tau}^{\varepsilon, k}(\phi)$ vanishes as $\varepsilon \rightarrow 0$, we have, by Kolmogorov's Lemma, that

$$
\begin{aligned}
& \lim _{\varepsilon \rightarrow 0} E^{\varepsilon}\left(\sup _{0 \leqq \tau \leqq T} \mid X_{\tau}^{\varepsilon, k}(\phi)-X_{0}^{\varepsilon, k}(\phi)-\int_{0}^{\tau} d \sigma\left[X_{\sigma}^{\varepsilon, k}\left(h ; \phi^{\prime \prime}\right)\right.\right. \\
& \quad+\phi^{\prime}(1)\left((k-1) h_{M-k}\left(\eta_{\varepsilon^{-2} \sigma}\right)-k h_{M-k+1}\left(\eta_{\varepsilon^{-2} \sigma}\right)\right) \\
& \left.\left.\quad+\phi^{\prime}(-1)\left(k h_{-M+k-1}\left(\eta_{\varepsilon^{-2} \sigma}\right)-(k-1) h_{-M+k}\left(\eta_{\varepsilon^{-2} \sigma}\right)\right)\right] \mid\right)=0,
\end{aligned}
$$

uniformly in $k \in \mathbb{Z}^{+}$. Hence, we also have, for each $k \in \mathbb{Z}^{+}$, that

$$
\begin{aligned}
& \lim _{\varepsilon \rightarrow 0} E^{\varepsilon}\left(\sup _{0 \leqq \tau \leqq T} \mid \tilde{X}_{\tau}^{\varepsilon, k}(\phi)-\tilde{X}_{0}^{\varepsilon, k}(\phi)-\int_{0}^{\tau} d \sigma\left[\tilde{X}_{\sigma}^{\varepsilon, k}\left(h ; \phi^{\prime \prime}\right)\right.\right. \\
& +\phi^{\prime}(1) \frac{1}{k} \sum_{m=R}^{k-1} \frac{1}{m} \sum_{j=R}^{m-1}\left((j-1) h_{M-j}\left(\eta_{\varepsilon^{-2} \sigma}\right)-j h_{M-j+1}\left(\eta_{\varepsilon^{-2} \sigma}\right)\right) \\
& \left.\left.+\phi^{\prime}(-1) \frac{1}{k} \sum_{m=R}^{k-1} \frac{1}{m} \sum_{j=R}^{m-1}\left(j h_{-M+j-1}\left(\eta_{\varepsilon^{-2} \sigma}\right)-(j-1) h_{-M+j}\left(\eta_{\varepsilon^{-2} \sigma}\right)\right)\right] \mid\right)=0
\end{aligned}
$$

with

$$
\tilde{X}_{\tau}^{\varepsilon, k}(\phi) \equiv \frac{1}{k} \sum_{m=R}^{k-1} \frac{1}{m} \sum_{j=R}^{m-1} X_{\tau}^{\varepsilon, j}(\phi)
$$


In terms of the average $\bar{\mu}^{\varepsilon}$ of the initial measure $\mu^{\varepsilon}$ over the time-interval $\left[0, \varepsilon^{-2} T\right]$, we have the bound

$$
\begin{aligned}
& E^{\varepsilon}\left(\sup _{0 \leqq \tau \leqq T} \mid \int_{0}^{\tau} d \sigma \frac{1}{k} \sum_{m=R}^{k-1} \frac{1}{m} \sum_{j=R}^{m-1}\left[\varepsilon \sum_{x=-\varepsilon^{-1}+j}^{\varepsilon^{-1}(1-l)} \phi^{\prime \prime}(\varepsilon x)\left(h_{x}\left(\eta_{\varepsilon^{-2} \sigma}\right)-\hat{h}\left(\frac{\varepsilon^{2}}{l} \sum_{y=x}^{x+\varepsilon^{-1} l} \eta_{y, \varepsilon^{-2} \sigma}\right)\right)\right.\right. \\
& \quad+\phi^{\prime}(1)\left((j-1) h_{M-j}\left(\eta_{\varepsilon^{-2} \sigma}\right)-j h_{M-j+1}\left(\eta_{\varepsilon^{-2} \sigma}\right)+\hat{h}\left(\rho_{+}\right)\right) \\
&\left.\left.\quad+\phi^{\prime}(-1)\left(j h_{-M+j-1}\left(\eta_{\varepsilon^{-2} \sigma}\right)-(j-1) h_{-M+j}\left(\eta_{\varepsilon^{-2} \sigma}\right)-\hat{h}\left(\rho_{-}\right)\right)\right] \mid\right) \\
& \leqq T \bar{\mu}^{\varepsilon}\left(\left|\frac{1}{k} \sum_{m=R}^{k-1} \frac{1}{m} \sum_{j=R}^{m-1} \varepsilon \sum_{x=-\varepsilon^{-1}+j}^{\varepsilon^{-1}(1-l)} \phi^{\prime \prime}(\varepsilon x)\left(h_{x}(\eta)-\hat{h}\left(\frac{\varepsilon}{l} \sum_{y=x}^{x+\varepsilon^{-1} l} \eta_{y}\right)\right)\right|\right. \\
&+\left|\phi^{\prime}(1)\left(\frac{1}{k} \sum_{m=R}^{k-1}\left(h_{M-m+1}-\hat{h}\left(\rho_{+}\right)\right)-\frac{2}{k} \sum_{m=R}^{k-1} \frac{1}{m} \sum_{j=R}^{m-1}\left(h_{M-j}-\hat{h}\left(\rho_{+}\right)\right)\right)\right| \\
&\left.+\left|\phi^{\prime}(-1)\left(\frac{-1}{k} \sum_{m=R}^{k-1}\left(h_{-M+m-1}-\hat{h}\left(\rho_{-}\right)\right)+\frac{2}{k} \sum_{m=R}^{k-1} \frac{1}{m} \sum_{j=R}^{m-1}\left(h_{-M+j}-\hat{h}\left(\rho_{-}\right)\right)\right)\right|\right) \\
&+O\left(\frac{R}{k}\right),
\end{aligned}
$$

using the summation by parts identity

$\frac{1}{m} \sum_{j=R}^{m-1} j\left(h_{ \pm(M-j)}-h_{ \pm(M-j+1)}\right)=\mp\left(\frac{1}{m} \sum_{j=R}^{m-1} h_{ \pm(M-j)}+h_{ \pm(M-j+1)}-\frac{R}{m} h_{ \pm(M-R+1)}\right)$.

We see that this upper bound gives zero as first $\varepsilon \rightarrow 0$ and then $k \rightarrow \infty$, by using Proposition 1 and 4 and as well that

$$
\lim _{k \rightarrow \infty} \lim _{\varepsilon \rightarrow 0} \bar{\mu}^{\varepsilon}\left(\left|\frac{1}{k} \sum_{j=R}^{k-1} h_{ \pm(M-j)}-\hat{h}\left(\rho_{ \pm}\right)\right|^{2}\right)=0,
$$

which follows from Proposition 4 and the argument used to establish Proposition 1. We infer that

$$
\begin{gathered}
E^{*}\left(\sup _{0 \leqq \tau \leqq T} \mid X_{\tau}(\phi)-X_{0}(\phi)-\int_{0}^{\tau} d \sigma\left[\int_{-1}^{1} d q \phi^{\prime \prime}(q) \hat{h}(\rho(q, \sigma))\right.\right. \\
\left.\left.-\phi^{\prime}(1) \hat{h}\left(\rho_{+}\right)+\phi^{\prime}(-1) \hat{h}\left(\rho_{-}\right)\right] \mid\right)=0 .
\end{gathered}
$$

This implies immediately the main statement (3.5) of (2).

The regularity $\rho \in L^{2}\left([0, T], H_{0}^{1}+\bar{\rho}\right)$ in the support of $P^{*}$ can be inferred from

$$
\begin{gathered}
E^{*}\left(\int_{0}^{T} d \tau \int_{-1}^{1} d q\left(\rho^{\prime}(q, \tau)\right)^{2}\right) \leqq \text { const. } T, \\
\lim _{l \rightarrow 0} E^{*}\left(\int_{0}^{T} d \tau\left(\frac{1}{l} \int_{1-l}^{1} d q \rho(q, \tau)-\rho_{+}\right)^{2}\right)=0,
\end{gathered}
$$


and

$$
\lim _{l \rightarrow 0} E^{*}\left(\int_{0}^{T} d \tau\left(\frac{1}{l} \int_{-1}^{-1+l} d q \rho(q, \tau)-\rho_{-}\right)^{2}\right)=0
$$

which are the consequences of Lemma 4 for a general $\mu^{\varepsilon} \in S(\varepsilon)$.

Finally, we complete the proof of Theorem 2 by demonstrating the uniqueness of weak solutions of (3.5) under the above regularity assumptions. Define $R$, for any $\rho \in L_{1}^{\infty}([-1,1])$, by

$$
R(x, y)=\int_{x}^{y} d q \rho(q)
$$

We introduce the notation $(F, G)=\int_{-1}^{1} d x \int_{-1}^{1} d y F(x, y) G(x, y)$. Then, if $\rho \in D\left([0, T], \mathscr{M}_{1}\right)$, $\Phi \in C^{1}\left([-1,1]^{2}\right)$, and $R(\tau)$ is associated to $\rho_{\tau}(q) \equiv \rho(q, \tau)$ via (3.37), it follows that

$$
\begin{aligned}
(R(\tau), \Phi) & =\int_{-1}^{1} d x \int_{-1}^{1} d y\left[\int_{x}^{y} d q \rho(q, \tau)\right] \Phi(x, y) \\
& =\int_{-1}^{1} d q \rho(q, \tau) \phi(q),
\end{aligned}
$$

with

$$
\phi(q) \equiv \int_{-1}^{q} d x \int_{q}^{1} d y \Phi(x, y)-\int_{q}^{1} d x \int_{-1}^{q} d y \Phi(x, y) .
$$

Observe that $\phi \in C^{2}([-1,1]), \phi( \pm 1)=0$ and

$$
\phi^{\prime}(q)=\int_{-1}^{1} d y \Phi(q, y)-\int_{-1}^{1} d x \Phi(x, q)
$$

If $\rho$ is in the support of $P^{*}$, it then follows that

$$
\begin{aligned}
(R(\tau), \Phi)= & \int_{-1}^{1} d q \phi(q) \rho_{0}(q) \\
& +\int_{0}^{\tau} d \sigma\left[\int_{-1}^{1} d q \phi^{\prime \prime}(q) \hat{h}(\rho(q, \sigma))+\phi^{\prime}(-1) \hat{h}\left(\rho_{-}\right)-\phi^{\prime}(1) \hat{h}\left(\rho_{+}\right)\right] \\
= & \int_{-1}^{1} d q \phi(q) \rho_{0}(q)-\int_{0}^{\tau} d \sigma \int_{-1}^{1} d q \phi^{\prime}(q) \hat{h}(\rho)^{\prime}(q, \sigma)
\end{aligned}
$$

by an integration by parts and the regularity assumption on $\rho$. Exploiting (3.40) gives finally

$$
(R(\tau), \Phi)=\left(R_{0}, \Phi\right)+\int_{0}^{\tau} d \sigma \int_{-1}^{1} d x \int_{-1}^{1} d y \Phi(x, y)\left[\hat{h}(\rho)^{\prime}(y, \sigma)-\hat{h}(\rho)^{\prime}(x, \sigma)\right]
$$

i.e.

$$
R(x, y ; \tau)=R_{0}(x, y)+\int_{0}^{\tau} d \sigma\left[\hat{h}(\rho)^{\prime}(y, \sigma)-\hat{h}(\rho)^{\prime}(x, \sigma)\right]
$$


in the $L^{2}$-sense. Clearly, $R(\tau)$ is absolutely continuous in $\tau$ as a map into $L^{2}\left([-1,1]^{2}\right)$ and

$$
\frac{\partial}{\partial \tau} R(x, y ; \tau)=\hat{h}(\rho)^{\prime}(y, \tau)-\hat{h}(\rho)^{\prime}(x, \tau), \quad \text { a.e. } \quad \tau \in[0, T],
$$

with identity in the $L^{2}$ sense.

Now consider for two weak solutions $\rho_{1}$ and $\rho_{2}$ of (3.5) the quantity

$$
W(\tau)=R_{1}(\tau)-R_{2}(\tau) .
$$

From what we have shown above it follows that

$$
\begin{aligned}
\frac{d}{d \tau}\|W(\tau)\|_{2}^{2}= & 2 \int_{-1}^{1} d x \int_{-1}^{1} d y\left(R_{1}(x, y ; \tau)-R_{2}(x, y ; \tau)\right) \\
& \cdot\left[\left(\hat{h}\left(\rho_{1}\right)^{\prime}(y, \tau)-\hat{h}\left(\rho_{2}\right)^{\prime}(y, \tau)\right)-\left(\hat{h}\left(\rho_{1}\right)^{\prime}(x, \tau)-\hat{h}\left(\rho_{2}\right)^{\prime}(x, \tau)\right)\right] \\
= & -8 \int_{-1}^{1} d y\left(\rho_{1}(y, \tau)-\rho_{2}(y, \tau)\right)\left(\hat{h}\left(\rho_{1}(y, \tau)\right)-\hat{h}\left(\rho_{2}(y, \tau)\right)\right) \leqq 0 .
\end{aligned}
$$

the latter equality obtained by an integration by parts and the inequality by the monotonicity of $\hat{h}$. Since $W(0)=0$, we conclude that for all $\tau \in[0, T]$

$$
W(\tau)=0
$$

in an $L^{2}$-sense; in particular, for all $\tau \in[0, T]$, a.e. $(x, y) \in[-1,1]^{2}$,

$$
\int_{x}^{y} d q\left[\rho_{1}(q, \tau)-\rho_{2}(q, \tau)\right]=0 .
$$

Therefore, $\rho_{1}=\rho_{2}$.

This concludes the proof of Theorem 2 .

\section{References}

[DIPP] DeMasi, A., Ianiro, N., Pellegrinotti, A., Presutti, E.: A survey of the hydrodynamical behavior of many-particle systems. In: Nonequilibrium phenomena II. Lebowitz, J. L., Montroll, E. W. (eds.), pp. 123-294. Amsterdam: North-Holland 1984

[ELS] Eyink, G., Lebowitz, J. L., Spohn, H.: Hydrodynamics of stationary nonequilibrium states for some stochastic lattice gas models. Commun. Math. Phys. 132, 253-283 (1990)

[GKMP] Galves, A., Kipnis, C., Marchioro, C., Presutti, E.: Nonequilibrium measures which exhibit a temperature gradient: Study of a model. Commun. Math. Phys. 81, 127-148 (1981)

[G] Georgii, H.-O.: Gibbs measures and phase transitions. Berlin, New York: Walter de Gruyter 1988

[GPV] Guo, M. Z., Papanicolaou, G. C., Varadhan, S. R. S.: Nonlinear diffusion limit for a system with nearest neighbor interactions. Commun. Math. Phys. 118, 31-59 (1988)

[M] Mitoma, I.: Tightness of probability measures on $C\left([0,1], S^{\prime}\right)$ and $D\left([0,1], S^{\prime}\right)$. Ann. Probl. 11, 989-999 (1983)

[Sp] Spohn, H.: Large scale dynamics of interacting particles. To appear in Texts and Monographs in Physics. Berlin, Heidelberg, New York: Springer 1990 
